\title{
Questes
}

\section{Obsolescence d'un personnage arborescent : quêtes autour de Gauvain}

Jessy Simonini

\section{(2) OpenEdition}

Édition électronique

URL : http://journals.openedition.org/questes/5226

DOI : 10.4000/questes.5226

ISSN : 2109-9472

Éditeur

Les Amis de Questes

Édition imprimée

Date de publication : 28 février 2019

Pagination : 117-130

ISSN : 2102-7188

\section{Référence électronique}

Jessy Simonini, «Obsolescence d'un personnage arborescent : quêtes autour de Gauvain », Questes

[En ligne], 40 | 2019, mis en ligne le 19 avril 2019, consulté le 22 avril 2019. URL : http:// journals.openedition.org/questes/5226 ; DOI : 10.4000/questes.5226 


\title{
Obsolescence d'un personnage arborescent : quêtes autour de Gauvain
}

\author{
Jessy SIMONINI \\ École Normale Supérieure de Paris
}

La lecture des principaux ouvrages de la matière arthurienne, et en particulier des romans en vers, confronte le lecteur à une grande variété de personnages, dont la nature et l'individualité ne peuvent pas être inscrites dans un schéma fixe mais qui, au contraire, évoluent selon leur contexte culturel et narratif. Ces personnages sont «omniprésents », pour utiliser la définition de Keith Busby dans son riche travail sur le personnage de Gauvain ${ }^{1}$. Ils sont donc le miroir d'un changement de sensibilité littéraire. Leur éventuelle obsolescence semble être celle du genre romanesque et, peut-être, d'un système de valeurs qui sont mises en doute et semblent être arrivées, au moins en apparence, à leur « automne ${ }^{2}$ ». Un extrait de La Mort du roi Arthur peut nous le montrer :

Ensi parlerent cil de Gaunes de la bataille; et moult s'esmerveillierent conment mé sire Gavains ot tant enduré, car tout savaient bien que Lanselos estoit li mieudres chevaliers del monde, et plus jouenes que mé sire Gavains n'estoit entour .XXI. an. Et a cele ore pooit bien avoir mé sire Gavains .LXXVI. ans, et li rois Artus .IIII.XX. ans et .XII. ${ }^{3}$.

\footnotetext{
${ }^{1}$ Keith Busby, Gauvain in Old French Literature, Amsterdam, Rodopi, 1980, p. 1.

${ }^{2}$ Johan Huizinga, L’Automne du Moyen Âge [1919], trad. Julia Bastin, Paris, Payot, 2015. À propos du déclin de la chevalerie, nous signalons Nicolas Le Roux, Le Crépuscule de la chevalerie. Noblesse et guerre au siècle de la Renaissance, Ceyzérieu, Champ Vallon, 2015.

${ }^{3}$ La Mort du roi Arthur, dans Le Livre du Graal, éd. Daniel Poirion, Paris, Gallimard, coll. «Bibliothéque de la Pléiade», 2009, t. 3, p. 1421. "C'est ainsi que ceux de Gaunes parlèrent du duel et ils s'étonnèrent beaucoup de l'endurance de monseigneur Gauvain car ils savaient bien que Lancelot était le meilleur chevalier du monde, et qu'il avait environ vingt-
} 
Le vieillissement physique des personnages peut correspondre à l'obsolescence du personnage littéraire, un personnage «épuisé », au seuil de son dernier « automne ». Cet épuisement correspond, dans ce cas particulier, à la mort d'Arthur et de Gauvain et, surtout, à l'effondrement définitif du royaume de Logres dans le cycle arthurien en prose. De son côté, Paul Zumthor a mis en évidence l'oubli irréparable du personnage de Merlin et, plus généralement, de la tradition médiévale :

La légende de Merlin, peut-on dire d'une certaine manière, est en voie d'extinction. [...] Strictement parlant $[\ldots]$ on peut ne voir là qu'un phénomène essentiellement provisoire : la culture contemporaine n'a, dans ses moyens d'expression, pas besoin de Merlin. En fait, le personnage, ses aventures, sont tombés dans un singulier oubli. Les œuvres de l'âge précédent n'ont pas survécu"

Il est légitime de se demander si l'«extinction» évoquée par Paul Zumthor en 1945 s'est vérifiée ou si, au contraire, sa prévision n'a pas été assez clairvoyante, si la littérature postérieure n'a pas suivi un autre chemin. Ce recommencement de la tradition littéraire médiévale, pour utiliser les mots d'Eugène Vinaver ${ }^{5}$, s'exprime dans une révérence particulière à l'héritage médiéval dans l'espace littéraire contemporain, mais aussi dans le cinéma, dans le théâtre et dans l'art en général.

En réalité, la fermeture d'un cycle romanesque et l'apparent épuisement d'un personnage sont toujours provisoires: le vieillissement n'est pas inéluctable, du moins dans le champ littéraire. Cette évidence est particulièrement frappante dans la culture et dans la littérature contemporaines, qui cherchent à réécrire les mythes littéraires du Moyen Âge pour trouver dans

et-un ans de moins que monseigneur Gauvain. À cette époque, monseigneur Gauvain pouvait être âgé de soixante-seize ans, et le roi Arthur de quatre-vingt-douze ans ».

${ }^{4}$ Paul Zumthor, Merlin le Prophète. Un thème de la littérature polémique, de l'historiographie et des romans, Genève, Slatkine, 1973, p. 287-288.

${ }^{5}$ Eugène Vinaver, À la recherche d'une poétique médiévale, Paris, Nizet, 1970, p. 138 : « tout commencement est un recommencement, rien ne saurait être négligé de ce qui le précède ou de ce qui pourra un jour venir s'ajouter ». 
ces topoï une nouvelle forme de vie ${ }^{6}$ et d'expression. De cette façon, des personnages disparus ou restés enfermés dans l'univers fixe et rigide du monde arthurien et de la culture européenne médiévale, appréhendés seulement par les recherches de philologues et de critiques désireux de les replacer dans un cadre théorique, retrouvent une nouvelle force et un nouveau sens. C'est par exemple le cas sous la plume de Jacques Roubaud, défini selon Baptiste Franceschini ${ }^{7}$ par l'heureuse expression d' « oulipien translateur ». Les histoires arthuriennes, cette matière médiévale riche et vaste, font ainsi l'objet d'une récriture contemporaine qui cherche à les sauver, à les soustraire à l'oubli par une opération de remémoration qui nous paraît rénovatrice et qui s'exprime dans plusieurs œuvres de Roubaud $^{8}$, mais aussi dans celles d'un autre membre de l'OuLiPo, l'Italien Italo Calvino.

Calvino en Italie et Roubaud en France sont deux excellents exemples du fait que la tradition médiévale ne s'est pas épuisée avec la fin du cycle arthurien et l'effondrement du royaume de Logres, mais que ses personnages, ses lieux et ses histoires ont survécu, comme part d'un imaginaire littéraire qui a franchi le seuil du $\mathrm{XX}^{\mathrm{e}}$ siècle et qui a donné vie à de nouvelles quêtes, de nouvelles formes de narration et de vie.

Mais quelles sont donc les formes de l'«automne » de Gauvain sur le plan littéraire? Pour Gaston Paris, le personnage de Gauvain est un «modèle

\footnotetext{
${ }^{6}$ Le concept de «forme de vie» a été repris et théorisé par le philosophe Ludwig Wittgenstein, dont le médiéviste Jacques Roubaud est un connaisseur (et que sa femme, Alix Cléo Roubaud, a étudié), comme le montre Jean-François Puff dans son Mémoire de la mémoire. Jacques Roubaud et la lyrique médiévale, Paris, Champion, 2009.

${ }^{7}$ La thèse de Baptiste Franceschini sur Jacques Roubaud «oulipien translateur» est une recherche très utile pour définir la nature de médiéviste de Roubaud, ses lectures et son rapport avec la tradition du Moyen Âge. Baptiste Franceschini, L'Oulipien translateur. La bibliothèque médiévale de Jacques Roubaud, Thèse en Littératures soutenue à l'université Michel-de-Montaigne - Bordeaux-III, sous la direction de Danièle James-Raoul et Francis Gingras, 2013.

${ }^{8}$ À ce sujet, voir Jacques Roubaud médiéviste, dir. Nathalie Koble et Mireille Séguy, Paris, Champion, 2018.
} 
accompli ${ }^{9} »$ de perfection et de chevalerie, tout en étant «dépourvu d'individualité $^{10} »$. Ces deux formules, qui peuvent être remises en question si l'on analyse plus en profondeur la nature et l'identité du personnage, nous aideront à observer comment se manifestent, au fil des textes arthuriens, le vieillissement de Gauvain en tant que chevalier et son obsolescence en tant que personnage sériel ${ }^{11}$.

\section{Vieillissement ou noircissement ?}

Comme le remarque avec pertinence Emmanuèle Baumgartner, « Gauvain est le héros toujours disponible d'une aventure qui commence ${ }^{12} »$; on peut le retrouver dans une pluralité de textes ${ }^{13}$, comme protagoniste de nombreuses aventures qui appartiennent à différentes traditions littéraires, et il est au centre d'aventures qui se multiplient et se ramifient dans une structure arborescente. Le récit de ses exploits n'a donc pas de conclusion définie mais reste ouvert, comme suspendu, en attente qu'une nouvelle aventure ou qu'une nouvelle histoire se matérialise. Baumgartner parle de «reconte ${ }^{14} »:$ une formulation qui montre bien la nature complexe et stratifiée de l'identité de Gauvain au fil des textes arthuriens et qui reflète la nature sérielle de ce personnage.

On sait que la véritable entrée en littérature du neveu d'Arthur se produit à la fin du XII ${ }^{\mathrm{e}}$ siècle, dans les romans de Chrétien, où il est le protagoniste d'une

\footnotetext{
${ }^{9}$ Gaston Paris, Histoire littéraire de la France, Paris, Imprimerie nationale, 1888, t. XXX, p. 33 .

${ }^{10}$ Ibid.

${ }^{11}$ Sur le thème de la sérialité du personnage, voir Richard Saint-Gelais, Fictions transfuges, Paris, Éditions du Seuil, 2011, mais aussi le récent essai de Thomas Conrad, Poétique des cycles romanesques de Balzac à Volodine, Paris, Classiques Garnier, 2016.

${ }^{12}$ Emmanuèle Baumgartner, «Les techniques narratives dans le roman en prose », dans Norris J. Lacy et alii, The Legacy of Chrétien de Troyes, Amsterdam, Rodopi, 1987, t. I, p. $167-190$.

${ }^{13}$ Dans les romans de Chrétien de Troyes, ainsi que dans les continuations du Conte du Graal (en particulier dans la première, qui lui est entièrement consacrée) et dans des romans arthuriens plus tardifs, notamment L'Âtre périlleux.

${ }^{14}$ Emmanuèle Baumgartner, «Les techniques narratives dans le roman en prose », art. cit., p. 169.
} 
évolution remarquable, d'une remise en question de ses valeurs et de ses traits caractéristiques, qui ont souvent poussé les critiques à y noter une forme de «déclin ${ }^{15}$. Dans notre perspective, ce noircissement pourrait aussi être lu comme une forme de vieillissement du chevalier.

Il est assez difficile de parler de vieillissement dans l'espace littéraire arthurien, où les coordonnées spatio-temporelles sont bien floues et difficiles à définir. Il est presque impossible, au moins pour le roman en vers, de trouver des traces précises de vieillissement des personnages principaux, sauf à de très rares exceptions. Dans le Conte du Graal, dernier roman de Chrétien, certains éléments obscurs, et probablement de caractère religieux, évoquent par exemple l'âge du roi Arthur ${ }^{16}$. Mais dans les romans versifiés, l'âge de Gauvain n'est pas précisé et il serait peu rigoureux d'identifier le déclin de Gauvain, qui se manifeste au fil du récit, comme un signe certain de son vieillissement. Il est cependant légitime de se demander si le noircissement du personnage de Gauvain et son apparent déclin peuvent constituer la trace d'une sénescence narrative.

Le noircissement de l'identité de Gauvain, son évolution imprévue d'un roman à un autre, d'un épisode à un autre, trouvent leur point de départ dans Erec et Enide, le premier roman de Chrétien, où Gauvain est qualifié par une formule qui restera célèbre et qui sera notamment utilisée au $\mathrm{Xx}^{\mathrm{e}}$ siècle par Roubaud dans la construction de son «axiomatique Gauvain ${ }^{17} »$. Cette formule pourrait fixer les traits d'un modèle hiératique de chevalerie, que Gauvain semble incarner mieux que tous les autres chevaliers de son époque : «devant

\footnotetext{
${ }^{15}$ Telle est l'idée véhiculée par Keith Busby, entre autres. Il serait néanmoins intéressant de proposer une nouvelle réflexion critique sur ce personnage en utilisant les catégories de transfictionnalité et de sérialité. À ce sujet, voir Richard Saint-Gelais, Fictions transfuges, op. cit., 2011.

${ }^{16}$ Ygerne, mère d'Arthur, en reprenant un sermon d'Isaïe, dit qu'Arthur tout à la fois a cent ans et n'est qu'un enfant : "Qu'il est anfes, li rois Artus, /s'il a .C. anz il n'a mie plus /ne plus ne puet il pas avoir » (Chrétien de Troyes, Le Conte du Graal, éd. Charles Méla, Paris, Le Livre de Poche, coll. «Lettres Gothiques », 1990, v. 8087-8089).

${ }^{17}$ Chapitre de la «fiction théorique » de Jacques Roubaud, Graal fiction, Paris, Gallimard, 1978.
} 
toz les buens chevaliers /doit estre Gauvains li premiers ${ }^{18} \gg$. Gauvain est le premier de tous les chevaliers, il possède une sorte de « privilège mythique » par rapport aux autres chevaliers. Cependant, dans Erec, il reste un personnage secondaire, tout en jouant un rôle remarquable dans la cour car il est le premier conseiller d'Arthur. Dans les premiers ouvrages de Chrétien, Gauvain n'a rien à démontrer : sa renommée parle à sa place et il n'a pas besoin de chercher des aventures pour valider son statut, qui ne semble pas être mis en cause. Il est le «plus vaillant, plus hardi, plus preu », « li preuz, li alosez ${ }^{19} »$, un modèle de chevalerie, de «mesure » et de compagnonnage possédant une autorité que personne ne peut mettre en question et qui en fait, à plusieurs reprises, le parangon de toute la classe chevaleresque. Il peut donc apparaître comme un personnage assez hiératique et fixe, ce qui pousse Gaston Paris à le considérer, on l'a vu, comme « dépourvu d'individualité ${ }^{20} »$.

Pourtant, dans les romans de Chrétien de Troyes, Gauvain est le protagoniste d'une évolution qui ne transforme pas radicalement sa nature, mais qui annonce un détournement de perspective, un nouveau regard. On passe du personnage modèle de chevalerie, fixé dans son rôle narratif (tel qu'il apparaît dans le Cligès et dans Erec et Enide, semblant en partie simplement traduire les formules des chroniques anglaises du XII siècle et du Roman de Brut) à un Gauvain « obscurci », un Gauvain «vieilli », soumis à de possibles échecs : telle est l'image qu'il revêt dans les deux derniers ouvrages de Chrétien, mais aussi dans quelques passages du Chevalier de la Charrette. Ainsi, par exemple, lorsqu'il tombe dans une rivière :

Et tantos qu'il viennent au pont

Ont mon seigneur Gauvain veü, del pont trabuchié et cheü

\footnotetext{
${ }^{18}$ Chrétien de Troyes, Erec et Enide, éd. Jean-Marie Fritz, Paris, Le Livre de Poche, coll. «Lettres gothiques », 1992, v. 5106-5109 et v. 1687-1688.

${ }^{19}$ Chrétien de Troyes, Cligès, éd. Méla-Collet, Paris, Le Livre de Poche, coll. «Lettres gothiques », 1994, v. 4853.

${ }^{20}$ Gaston Paris, Histoire littéraire de la France, op. cit., t. XXX, p. 33.
} 
An l'eve qui estoit parfonde

[...]

Mes ne cuident pas que il vive

cil qui l'ont tret de l'eve fors, car il en avoit molt el cors ${ }^{21}$.

Les hommes de Lancelot se trouvent face à un Gauvain en difficulté : le chevalier, qui devrait les aider à retrouver Lancelot, semble mort. Dépourvu de son « hauberc » et de son «hiaume» (v. 5114-5115), épuisé et souffrant dans l'épreuve du passage par le pont sous l'eau (alors que Lancelot avait choisi le pont de l'épée), Gauvain est dépeint sous une lumière profondément humaine. C'est en effet sur son humanité que Chrétien met l'accent en montrant, à travers une scène qui possède aussi des traits comiques, que le « soleil de la chevalerie » est un chevalier comme les autres, soumis à des défaillances remarquables.

Keith Busby souligne que «Chrétien cherche à ne pas représenter Gauvain sous une lumière trop négative » et qu'il ne s'agit pas d'un «Don Quichotte avant la lettre, mais d'un noble et vaillant chevalier qui souffre d'une momentanée perte de dignité ${ }^{22}$. Cette scène n'est pas une parodie du monde chevaleresque et des valeurs dont Gauvain reste l'un des modèles, et sa «perte de dignité » peut être lue comme l'acquisition d'un nouveau statut, plus humain, détaché de toute idéalisation. Ce nouveau statut caractérise aussi le développement du personnage de Gauvain dans la Continuation qui lui est presque entièrement consacrée et dans les ouvrages attribués à Raoul de Houdenc, ainsi que dans l'Âtre périlleux. On peut cependant considérer aussi cette perte comme un premier signe de sénescence.

Dans ce même roman, qui marque donc un passage important voire une fracture dans le développement du personnage de Gauvain, Lancelot s'interroge

\footnotetext{
${ }^{21}$ Le Chevalier de la Charrette, éd. Charles Méla, Paris, Le Livre de Poche, coll. « Lettres gothiques », 1992, v. 5106-5109: «et dès leur arrivée au pont,/ils ont vu monseigneur Gauvain /qui, ayant basculé, était tombé /dans l'eau, profonde à cet endroit. ».

22 «Chrétien is careful not to show Gauvain in a too unfavorable light», et aussi « Gauvain is not really a ridiculous Don Quichotte avant la lettre, but a noble, well-motivated knight suffering a temporary loss of dignity » (Keith Busby, Gauvain in Old French Literature, op. cit., p. 61-62).
} 
sur l'absence du chevalier, qui ne le rejoint pas pour le libérer de Baudemagu et Méléagant. Les lamentations de Lancelot prisonnier contribuent à assombrir l'image que l'on s'est construite de Gauvain, à travers la médiation d'une voix extérieure qui met en doute sa nature courtoise même :

Certes trop i par demorez si ne feites pas corteisie, bien deüst avoir vostre aïe cil cui tant soliez amer ${ }^{23}$.

Et encore :

Li vilains dit bien voir qu' a poinne puet an mes un ami trover, de legier puet an esprover as besoing qui est boens amis ${ }^{24}$.

Dans ces deux passages au discours direct, deux traits topiques du chevalier sont interrogés : sa courtoisie (il est accusé d'en manquer) et la fidélité de son amitié. L'absence de Gauvain, lorsque Lancelot est emprisonné, met forcément en question sa valeur et sa courtoisie. On peut noter encore une fois, dans ces vers, une double tension entre l'image topique de Gauvain et son comportement dans le développement narratif. Ces exemples, comme d'autres passages du Conte du Graal où l'image de Gauvain est plus noircie encore par ses échecs, peuvent nous montrer que ce personnage se caractérise, au plan fictionnel, par une profonde évolution et une remise en question de $l^{\prime}$ « axiomatique » de départ.

Il est légitime de se demander si c'est Gauvain, dont Chrétien ne met jamais en évidence l'âge, qui a vieilli, ou si c'est son image et sa représentation qui connaissent une obsolescence, au profit d'autres modèles de chevalerie incarnés par de nouvelles figures. Dans les romans en vers, en particulier ceux de Chrétien, les aventures des personnages ont lieu dans un univers spatiotemporel «autre». En dehors de toute temporalité, Chrétien et ses continuateurs

\footnotetext{
${ }^{23}$ Le Chevalier de la Charrette, éd. cit., v. 6488-6491.

${ }^{24}$ Ibid., v. 6502-6505.
} 
ne questionnent presque jamais l'âge des personnages, alors que l'extrait de la Mort du roi Arthur proposé dans l'introduction ouvre une nouvelle perspective sur ce sujet. Si, dans le Conte du Graal, la formulation d'Ygerne à propos de l'âge d'Arthur n'est pas limpide ${ }^{25}$, dans la Mort du roi Arthur, on retrouve une déclaration assez explicite concernant l'âge d'Arthur, de Gauvain et de Lancelot. Le monde arthurien, dans cet ouvrage, est plongé dans un véritable « automne », apparemment irréversible : «le calendrier festif des cours officielles, véritable armature de la chevalerie arthurienne, perd toute consistance», dit Philippe Walter, et ce déclin se lit «dans la disparition du calendrier traditionnel du récit ${ }^{26}$. La persistance des valeurs chevaleresques, vestiges d'un monde en disparition, s'accompagne d'une extrême dégradation morale des personnages, de ceux qui avaient été des «modèles » pour ces valeurs. Dans ce passage, l'obsolescence et le vieillissement des personnages arthuriens, qui s'articulent de façon parallèle au fil du récit, émergent avec une certaine force. Pour la première fois, l'auteur anonyme de la Mort Artu dit en effet que «cele ore pooit bien avoir mé sire Gavains .LXXVI. ans, et li rois Artus .IIII.XX. ans et .xii. ${ }^{27}{ }^{2}$. Le sentiment du temps se manifeste après avoir toujours été caché, au moins sur le plan narratif, et apparaît ici avec toute sa force et sa puissance, dans la violence d'une formule qui donne une image nouvelle d'Arthur et de Gauvain. En précisant l'âge des deux chevaliers, l'auteur de la Mort Artu introduit pour la première fois la possibilité de leur vieillissement et, donc, du passage du temps dans l'univers statique des héros arthuriens.

$\mathrm{Au}$ sein de l'ouvrage de clôture du cycle, l'obsolescence du personnage semble s'accompagner de son déclin physique. Le déclin passe, de cette façon, par une dégradation morale et par un vieillissement physique mis en évidence.

\footnotetext{
${ }^{25}$ Le Conte du Graal, éd. cit., v. 8087-8089.

${ }^{26}$ Philippe Walter, La Mémoire du temps. Fêtes et calendriers, de Chrétien de Troyes à la Mort Artu, Paris, Champion/Slatkine, 1989, p. 217.

${ }^{27}$ La Mort du roi Arthur, éd. cit., p. 1421 : «À cette époque, monseigneur Gauvain pouvait être âgé de soixante-seize ans, et le roi Arthur de quatre-vingt-douze ans ».
} 
Gauvain, du modèle de toute chevalerie, devient un modèle (ou, en l'occurrence, un contre-modèle) de démesure et, malgré son âge, il n'hésite pas à se battre contre un Lancelot bien plus jeune que lui.

La référence directe au vieillissement de Gauvain semble dans ce cas devoir transférer sur le plan thématique, dans une obsolescence temporelle, une obsolescence qui n'est initialement que littéraire et narrative. L'auteur, considérant Gauvain comme narrativement dépassé, décide de souligner symboliquement ce sentiment de lecture à travers une référence à son âge.

\section{Une naissance dans l'obsolescence}

Pour aborder la question de l'obsolescence de Gauvain sur le plan narratif, il faut se placer à un stade encore embryonnaire de la tradition écrite arthurienne, notamment dans les chroniques où Gauvain apparaît déjà comme un prototype de personnage sériel, passant d'une tradition à une autre, d'un nom à un autre ${ }^{28}$, comme s'il était déjà un «monument» à l'aune duquel les autres chevaliers devaient se mesurer. C'est ce que montre cet extrait d'une chronique anglaise datée du début du XII ${ }^{\mathrm{e}}$ siècle et attribuée à Guillaume de Malmesbury :

Tunc in provincia Walarum, quae Ros vocatur inventum est sepulchrum Walwen, qui fuit haud degener Arturis ex sorore nepos. Regnavit in ea parte Britanniae quae adhuc Walweitha vocatur: miles virtute nominatissimus, sed a fratre et nepote Hengistii, de quibus in primo libro dixi, regno expulsus, prius multo eorum detrimento exilium compensans suum; communicans merito laudi avunculi, quod ruentis patriae casum in plures annos distulerint. Sed Arturis sepulchrum nusquam visitur, unde antiquitas naeniarum adhuc eum venturum fabulatur ${ }^{29}$.

\footnotetext{
${ }^{28}$ Le Walwein des chroniques arthuriennes, le Gauvain des romans arthuriens, le Gawain de Sir Gawain and The Green Knight, roman en moyen anglais du XIV ${ }^{\mathrm{e}}$ siècle.

${ }_{29}$ «En ce temps-là, dans une province des Galles qui se nomme Ros fut découvert le tombeau de Walwen qui fut par sa mère le digne neveu d'Arthur. Il régna dans cette partie de la Bretagne appelée encore Walweitha: soldat d'une vertu illustre qui, chassé de son royaume par le frère et le neveu d'Hengeste, dont j'ai parlé au livre premier, vengea d'abord cet exil en
} 
Dans ce texte, Gauvain est déjà associé à «Arturis » dont il était le neveu maternel ( «ex sorore nepos»), une remarque qui est cohérente avec les représentations généalogiques successives de Gauvain dans les romans arthuriens. Surtout, l'évolution d'un personnage qu'on peut considérer à la fois comme réel et mythique passe par l'évocation de son sepulchrum, son tombeau. Cette chronique, dans laquelle Gauvain est probablement évoqué pour la première fois à l'écrit (dans l'une des nombreuses variations graphiques de son nom, «Walwen »), l'associe déjà à un passé mythique, à un tombeau qui témoigne de sa disparition et de sa possible résurgence symbolique et fictionnelle. Il est déjà un souvenir et une trace : celle-ci reste bien présente, dans la géographie et dans le paysage de la Bretagne, dans le royaume de «Walweitha» sur lequel, comme nous le raconte Guillaume de Malmesbury, régnait un «miles virtute nominatissimus ». Cette formule accompagne par ailleurs Gauvain tout au long du cycle arthurien, en particulier des romans en vers. Mais dans cet extrait, il est déjà représenté comme le vestige d'un monde qui n'existe plus que sous la forme d'un monument de pierre.

La naissance de Gauvain, à travers cette chronique, est une naissance dans l'obsolescence : l'évocation de ce personnage semble correspondre à celle d'un univers disparu et que l'on cherche à soustraire à l'oubli. La perspective de l'obsolescence pourrait alors être complètement renversée : ce tombeau est aussi le point de départ d'une opération de remémoration et d'écriture qui, d'un certain point de vue, semble «monumentaliser » le personnage de Gauvain, le transformer pour la première fois en un acteur possible de sérialité, constituant

leur infligeant de grosses pertes. Il mérite d'être associé à la gloire de son oncle, ayant soutenu comme lui pendant un certain nombre d'années la cause de sa patrie défaillante. Mais le tombeau d'Arthur ne se voit nulle part et c'est pourquoi de vieilles fables racontent qu'il reviendra » (Philippe Walter, Gauvain le chevalier solaire, Paris, Imago, 2013, p. 17). Voir également The History of the Kings of England and the Modern History of William of Malmesbury, éd. John Sharpe, London, W. Bulmer \& Co. for Longman, Hurst, Rees, Orme \& Brown, 1815, p. 358-359. 
d'un «espace littéraire ${ }^{30} »$. La transformation de Gauvain en «monument de l'imaginaire » part de son tombeau, d'un lieu qui témoigne de sa disparition, pour se développer dans une nouvelle dimension, comme trace vivante et objet d'une écriture qui invente le passé et qui trouve ses racines dans une trace obsolète. À l'autre bout de la chaîne littéraire médiévale, la catégorie du «monument » n'échappe pas à l'Arioste, qui l'introduit dans un passage de son Orlando furioso (1532) où il évoque une série de chevaliers «bretons » dont il reste encore des traces :

Gran cose in essa già fece Tristano, Lancillotto, Galasso, Artù e Galvano, Ed altri cavallieri e de la nuova E de la vecchia Tavola famosi :

Restano ancor di più d'una lor pruova Li monumenti e li trofei pomposi ${ }^{31}$.

Cette liste de noms qui se succèdent dans les vers du plus grand poème chevaleresque du XVI ${ }^{\mathrm{e}}$ siècle témoigne du même souci de «monumentalisation » et de son succès : les chevaliers sont «célèbres », «illustres », et ce qui reste d'eux n'est que «monuments ». Ce mot se prête donc à plusieurs significations : il pourrait s'agir de véritables monuments visuels, de traces sous forme de statues ou de tombeaux dans un paysage défini, mais aussi de monuments littéraires, traces écrites d'une tradition que les contemporains de l'Arioste maîtrisent et utilisent pour leurs propres écritures, comme source et comme parangon.

\footnotetext{
${ }^{30}$ Maurice Blanchot, L'Espace littéraire, Paris, Gallimard, 1955, p. 31. Pour Blanchot, «Écrire [...] c'est se livrer au risque de l'absence du temps, où règne le recommencement éternel ». L'association entre l'écriture et le recommencement, qui pour Blanchot doit être interprétée dans une autre direction, nous renvoie aussi à la question des récritures médiévales où toute récriture est un recommencement, comme nous l'avons déjà remarqué.

${ }^{31}$ L'Arioste, Orlando furioso, éd. Sanguineti-Turchi, Milano, Garzanti, 1964, chant IV, strophes 52-53 : «Tristan, Lancelot, Galaas, Artus, Gauvain, et tant d'autres chevaliers, l'orgueil de la Table-Ronde ancienne et nouvelle, l'ont rendue célèbre et y ont laissé des traces glorieuses de leurs exploits » (trad. : Roland furieux. Nouvelle traduction, avec la vie de L'Arioste, et des notes sur les romans chevaleresques, les traditions orientales, les chroniques, les chants des trouvéres et des troubadours comparés au poème de L'Arioste, Knab, 1839, vol. 1).
} 
Le vieillissement de Gauvain est préparé, au fil des textes, par un lent déclin qui remet en cause l'image de ce chevalier et en propose une autre, noircie. Ce déclin progressif aboutit à une fracture importante : la certitude du vieillissement de Gauvain - comme on peut l'observer dans le court passage de la Mort du roi Arthur, lorsqu'on a l'impression que le temps passé se matérialise brutalement en un seul instant, peu avant la mort du chevalier.

$\mathrm{Si}$, sur le plan narratif, un vieillissement peut être remarqué, l'obsolescence de Gauvain comme personnage littéraire et comme symbole d'une tradition est moins évidente. Le chevalier naît déjà dans l'obsolescence sous la forme d'un tombeau qui s'inscrit comme trace mémorielle dans le paysage, et il traverse les siècles et les traditions littéraires. Sa monumentalisation n'entraîne pas une forme d'obsolescence, mais bien au contraire le point de départ d'une arborescence narrative qui se manifeste aussi de nos jours, notamment sous la plume de Jacques Roubaud ${ }^{32}$ où Gauvain reprend vie et échappe à un nouvel oubli possible.

\footnotetext{
${ }^{32}$ Dans Graal Fiction, op. cit., par exemple, où Roubaud consacre un chapitre entier à Gauvain («Who’s who », p. 59-142).
} 
